\title{
Digesting omni-video along routes for navigation
}

\author{
Hongyuan Cai and Jiang Yu Zheng \\ School of Science
}

\begin{abstract}
Indiana University - Purdue University Indianapolis
\end{abstract}
\begin{abstract}
Omni-directional video records complete visual information along a route. Though replaying an omni-video presents reality, it requires significant amount of memory and communication bandwidth. This work extracts distinct views from an omni-video to form a visual digest named route sheet for navigation. We sort scenes at the motion and visibility level and investigate the similarity/redundancy of scenes in the context of a route. We use source data from 3D elevation map or omni-videos for the view selection. By condensing the flow in the video, our algorithm can generate distinct omni-view sequences with visual information as rich as the omni-video for further scene indexing and navigation with GIS data.
\end{abstract}

